\title{
Why are influenza vaccination coverage rates still too low among health care workers in a tertiary care children's hospital in Turkey?
}

\author{
Aysegul Ertugrul $^{1}$ (1) $\cdot$ Eyup Sari $^{2} \cdot$ Nazli Gulenc $^{2} \cdot$ Serap Ozmen $^{1}$ (1)
}

Published online: 10 December 2020

(c) Springer Nature Limited 2020

\begin{abstract}
Annual influenza vaccination offers the best means to control and prevent influenza-associated illnesses. Vaccination of health care workers (HCWs) is crucial for reducing influenza-related morbidity and mortality, and health authorities recommend universal vaccination of this population. We evaluate the rates of influenza vaccination among HCWs and factors influencing uptake among health care professional groups in a children's hospital in Ankara, Turkey. We surveyed $108 \mathrm{HCWs}$ using a self-administered questionnaire. Influenza vaccination coverage rates (VCR) among HCWs for at least one dose in their lifetimes was 60.2\%. In the 2016-2017 influenza season, VCR of HCWs was $14.8 \%$. We found a significantly higher rate of vaccinated participants among physicians $(83.3 \%)$, older HCWs (mean age \pm SD, $36.2 \pm 7.7$ year), HCWs who believed in the necessity for vaccination (79.5\%), and HCWs who have worked for a longer time ( $>5$ years) in the health care profession (71.2\%) ( $p=0.023, p=0.002, p=0.001, p=0.003$ respectively). For those who refused vaccination, the most common reason was doubts about its effectiveness. The most used source for information about the influenza vaccine among HCWs was the Ministry of Health (MoH). The MoH's policies should prioritize HCWs to improve VCRs.
\end{abstract}

Keywords Healthcare worker $\cdot$ Health policy $\cdot$ Influenza $\cdot$ Seasonal $\cdot$ Vaccination coverage $\cdot$ Attitude $\cdot$ Vaccine

\section{Abbreviations}

HCWs Health care workers

Türk Toraks Derneği Uluslararası Katılımlı 22. Yıllık Kongresi, 13 May 2019, Oral Presentation.

Electronic supplementary material The online version of this article (https://doi.org/10.1057/s4127 1-020-00250-1) contains supplementary material, which is available to authorized users.

Aysegul Ertugrul

aysegull.ertugrul@gmail.com

Extended author information available on the last page of the article 
VCR Vaccination coverage rates

$\mathrm{MoH} \quad$ Ministry of Health

\section{Introduction}

Influenza is a major public health problem. It occurs all over the world, with an annual attack rate estimated at $5-10 \%$ in adults and $20-30 \%$ in children [1]. Three worldwide outbreaks of influenza took place in the 20th century and gave rise to heavy social and healthcare burdens [2]. The world will face another influenza pandemic, but we do not know when, or how severe it will be.

The most cost-effective way to prevent the disease is influenza vaccination [3]. Annual influenza vaccination has proved to be the best way to control and prevent the burden of influenza-associated illnesses, hospitalization, mortality, and economic impact in the population. According to a recommendation of The United States (US) Advisory Committee on Immunisation Practices (ACIP), persons $\geq 6$ months should be vaccinated annually against influenza, preferably by the end of October. In the US, October is the start of colder weather. Recommended timing for the influenza vaccination may differ by hemisphere due to seasonality [4, 5]. Many international guidelines recommend vaccination for all health care workers (HCWs), but vaccination coverage rates (VCR) among $\mathrm{HCW}$ semain far below the targets [6]. At the United States Centers for Disease Control and Prevention (CDC), the National Health Interview Survey (NHIS) reported a VCR of $<40 \%$ among HCWs in its 2003 survey [7].

In Turkey, for individuals at risk (pregnant women, those with chronic diseases, elderly $>65$ years of age, children between 6 months and 18 years old who are on long term acetylsalicylic acid, people living in nursing homes and care centers for the elderly, and HCWs), the social security institution pays for the vaccine based on a recommendation of Ministry of Health $(\mathrm{MoH})$ [8]. The Turkish $\mathrm{MoH}$ has provided influenza vaccine to HCWs free of charge every year since 2002. In 2006, in a crosssectional study, physicians found that $37.1 \%$ of HCWs were considering vaccination [9]. Ozisik et al. [10] reported that the VCR against influenza among HCWs in Turkey to be between 14.5 and $45.7 \%$ in several studies, far below the rates reported from the US, but similar to Europe. In the US, 78.4\% of HCWs reported having been vaccinated against influenza during the 2017-2018 season, similar to coverage reported in the previous four influenza seasons [11]. In Europe, influenza VCRs for the 2012-2013 season among the HCWs varied widely, from 9.5 to $45.6 \%$ [12].

The influence of influenza vaccination among HCWs is multidirectional. HCWs are mentors who recommend vaccination and motivate patients' to comply, and they are a target group for the control of the infection. Specifically, vaccination of HCWs protects them from influenza infection during the season, decreases their risks of becoming unrecognized sources of infection, and prompts change in the behavior of their patients [10].

Despite many efforts to increase VCR, the number of anti-vaccine supporters in Turkey increases daily, as elsewhere in the world [13]. Anti-vaccination movements lower vaccination coverage. Those against vaccination easily communicate to the 
general public using the internet. HCWs are the most important connection between the community and scientific authorities. HCWs' advice and encouragement to the public is one of the best vaccine promotions. HCWs attitudes and health practices guide society and could influence many to help prevent the disease, but vaccination coverage of HCWs is low [14]. The World Health Organization (WHO) has identified vaccine hesitancy as one of the top 10 threats to global health in 2019 . WHO also highlighted the importance of health workers in communities, as they remain the most trusted advisors and influencers of vaccine implementation [15].

In this study, we aimed to evaluate the influenza VCRs among HCWs and the factors influencing influenza vaccination uptake among different health care professional groups at the Health Sciences University Dr Sami Ulus Maternity and Children Training and Research Hospital in Ankara, Turkey in 2017.

\section{Methods}

We conducted this study at the Health Sciences University Dr Sami Ulus Maternity and Children Training and Research Hospital in Ankara, Turkey during 3 months, from April 2017 through June 2017. We obtained approval from the Hospital's ethics committee. Although the Hospital has affiliates in several regions of Ankara, we limited our study to HCWs in one region. Our study sample included all HCWs in one region of the hospital except those who started working after 2015. We invited all of them to participate in the survey. The study population consisted of five groups; physicians, physician assistants, nursing staff, auxiliary medical staff, and administrative staff. All physicians and physician assistants participated in the survey were pediatricians.

In the sample $(n=163)$, we interviewed $72 \%$ of the HCWs and $92 \%$ completed survey. A physician's assistant delivered a paper questionnaire to HCWs in a closed envelope, then collected anonymous paper questionnaires on the same day. Participation was entirely voluntary. We asked all participants in this crosssectional survey to complete a self-administered questionnaire. We selected the questions based on previous studies that evaluated the knowledge and attitudes toward the influenza vaccine $[9,10,16,17]$. Prior to conducting the survey, the study team checked the questionnaire for unsuitable questions with assistance from five individuals for each profession group. We sought clarity to ease completion by participants and accuracy of answers. The questionnaire asked about knowledge, attitudes, and HCWs' practices involving influenza vaccination. We divided the instrument into four sections with a total of 27 questions:

Section 1: seven questions about sociodemographics: age, sex, profession, duration of working in the hospital, and medical conditions of themselves and their families.

Section 2: nine questions to assess vaccine administration practice; vaccination during the past years and observed side effects. 
Section 3: eight questions about vaccine knowledge of the HCWs; target groups for vaccine, timing and effectiveness of the vaccine, $\mathrm{MoH}$ recommendations about the vaccine.

Section 4: three questions about attitudes, reasons for acceptance or refusal of the vaccine, and their attitudes about the need for vaccination. These questions were close-ended with nominal or categorical (yes/no, multiple-choice list) responses.

The study team maintained privacy and confidentiality throughout the implementation of the survey and study period. Questionnaires included no identifiable information about research subjects.

We applied a Chi squared test to assess differences in categorical variables. We considered a $p$ value of $<0.05$ to be statistically significant. For the data set with missing items (6.5\%), we attempted available case analysis. We used Odds ratios and Cohen's $d$-effect size to assess the relationship. We analyzed all data using the statistical program SPSS (Statistical Package for Social Sciences 15; SPSS Inc., Chicago, IL, USA).

\section{Results}

A total of $108 \mathrm{HCW}$ completed the questionnaire (response rate 92\%), including $18(16.7 \%)$ physicians, $23(21.3 \%)$ physician assistants, $33(30.6 \%)$ nursing staff, $13(12 \%)$ auxiliary medical staff, 14 (13\%) administrative staff. For 7 (6.5\%) of all responses, data about profession were missing. Table 1 shows the baseline characteristics of the respondents who completed the survey.

Sixty-five HCWs $(60.2 \%)$ reported having been vaccinated for influenza at least once in their lifetimes and $43 \mathrm{HCWs}$ (39.8\%) reported to never having been

Table 1 Characteristics of study participants

\begin{tabular}{|c|c|c|c|c|c|}
\hline Characteristics & Physician $n(\%)$ & $\begin{array}{l}\text { Physician } \\
\text { assistant } n \\
(\%)\end{array}$ & $\begin{array}{l}\text { Nursing staff } n \\
(\%)\end{array}$ & $\begin{array}{l}\text { Auxiliary staff } \\
n(\%)\end{array}$ & $\begin{array}{l}\text { Adminis- } \\
\text { trative staff } \\
n(\%)\end{array}$ \\
\hline Total number & 18 & 23 & 33 & 13 & 14 \\
\hline $\begin{array}{l}\text { Age (year), } \\
\text { mean } \pm \text { SD }\end{array}$ & $36 \pm 7.6$ & $29.3 \pm 6$ & $34 \pm 7.4$ & $32.8 \pm 7.1$ & $35.5 \pm 10.9$ \\
\hline \multicolumn{6}{|l|}{ Sex } \\
\hline Female & $10(55.6)$ & $14(60.9)$ & $23(69.7)$ & $6(46.2)$ & $6(42.8)$ \\
\hline Male & $8(44.4)$ & $9(39.1)$ & $10(30.3)$ & $7(53.8)$ & $8(57.1)$ \\
\hline \multicolumn{6}{|c|}{ Years of working } \\
\hline$\leq 5$ years & $3(16.7)$ & $21(91.3)$ & $7(21.2)$ & $6(46.2)$ & $5(35.7)$ \\
\hline $6-10$ years & $4(22.2)$ & 0 & $6(18.2)$ & $3(23.1)$ & $2(14.3)$ \\
\hline$>10$ years & $11(61.1)$ & $2(8.7)$ & $20(60.6)$ & $4(30.8)$ & $7(50)$ \\
\hline \multicolumn{6}{|c|}{ Any chronic disease } \\
\hline No & $14(77.8)$ & $11(47.8)$ & $19(57.6)$ & $9(69.2)$ & $8(57.1)$ \\
\hline
\end{tabular}


vaccinated against influenza. The VCR of HCWs having H1N1 swine flu vaccine in 2009 was $29.9 \%$. (See reported VCRs by HCW groups in Supplemental Figure S1.) Physicians had the highest vaccination rate (83.3\%), significantly so. We also found significantly higher rates in older HCWs (mean age $\pm \mathrm{SD}, 36.2 \pm 7.7$ year), HCWs who believed in the necessity for vaccination $(79.5 \%)$, and HCWs who had worked for a longer time in the health care profession $(71.2 \%)(p=0.002, p=0.001$, $p=0.003$ respectively) (Table 2 ).

Vaccination in the 2016-2017 influenza season reached $14.8 \%$ of HCWs and the VCR of HCWs' household family members was $27.8 \%$. None of the respondents who received the vaccine in 2016-2017 influenza season reported having been severely ill with flu in the influenza season, however in the non-vaccinated group, $10.2 \%$ of them reported severe symptoms of flu.

Ninety participants $(83.3 \%)$ reported vaccine-associated side effects. Thirty-four of the 90 participants associated their side effects with the vaccine. Participants most commonly reported vaccine-associated side effect side effects as symptoms of cold or influenza, such as sore throat, runny nose or nasal congestion.

Resources HCWs used to learn about vaccines appear in Supplemental Figure S2. They relied most often on the Ministry of Health $(\mathrm{MoH})$ for information: $83.3 \%$ of the auxiliary medical staff, $66.7 \%$ of the nursing staff, $65.2 \%$ of the physician assistants, $50 \%$ of the physicians, and $50 \%$ of the administrative staff stated they turned to $\mathrm{MoH}$ for information about the influenza vaccine.

HCWs were most knowledgeable about MoH's recommendation to vaccinate all HCWs $(89.8 \%)$, the groups $\mathrm{MoH}$ recommended for vaccination $(72.2 \%)$, and timing recommended for vaccine administration (70\%). Respondents of each professional group, except auxiliary and administrative staff, exceeded the threshold of $70 \%$ correct answers for those questions. HCWs were least knowledgeable about the period for which influenza vaccine confers protection (17.8\%). Vaccine knowledge questionnaire responses appear in Table 3.

Seventy-four participants $(68.5 \%)$ provided reasons for refusing vaccination (Supplemental Figure S3). The most common reason for rejecting the vaccine was doubts about its effectiveness. Thirty-one participants $(28.7 \%)$ provided reasons for their willingness to receive the vaccine (Supplemental Figure S4). The most common reason given, "HCWs are at risk". Physicians' belief in necessity for vaccination was highest at $66.7 \%$, then: $65.2 \%$ for physician assistants, $28.6 \%$ for administrative staff, $23.1 \%$ for auxiliary staff and $21.2 \%$ for nursing staff.

\section{Discussion}

Influenza results in a substantial burden on health services and with serious socioeconomic effects worldwide [3]. The best way to prevent and control influenza infection is annual vaccination, but suboptimal VCR among target groups has remained a significant problem for many years [3]. Vaccination yields benefits both clinically and economically. In our sample, the overall VCR for influenza, at least once during a lifetime, was $60.2 \%$. However, $29.9 \%$ of the HCWs received H1N1 swine flu vaccine in 2009 , and $14.8 \%$ of the HCWs received the influenza vaccine in the 
Table 2 The characteristics of the participants according to their vaccination status

\begin{tabular}{|c|c|c|c|c|}
\hline & $\begin{array}{l}\text { Vaccinated at least once } \\
\text { ever with influenza } \\
\text { vaccine } \\
n(\%)\end{array}$ & $\begin{array}{l}\text { Never vaccinated } \\
\text { with influenza } \\
\text { vaccine } \\
n(\%)\end{array}$ & $p$ value & OR $(95 \% \mathrm{CI})$ \\
\hline $\begin{array}{l}\text { Total number of par- } \\
\text { ticipants }\end{array}$ & 65 & 43 & & \\
\hline Physician & $15(25)$ & $3(7.3)$ & 0.023 & $4.2(1.13-15.69)$ \\
\hline Other HCWs ${ }^{\mathrm{a}}$ & $45(75)$ & $38(92.7)$ & & \\
\hline Doctors $^{\mathrm{b}}$ & $27(45)$ & $14(34.1)$ & 0.275 & $1.5(0.694-3.589)$ \\
\hline Other HCWs $\mathrm{s}^{\mathrm{c}}$ & $33(55)$ & $27(65.9)$ & & \\
\hline \multicolumn{5}{|l|}{ Sex } \\
\hline Female & $40(61.5)$ & $24(55.8)$ & 0.553 & $0.789(0.361-1.726)$ \\
\hline Male & $25(38.5)$ & $19(44.2)$ & & \\
\hline Age(year), mean \pm SD & $36.2 \pm 7.7$ & $30.7 \pm 10$ & 0.002 & $0.612^{\mathrm{d}}$ \\
\hline \multicolumn{5}{|l|}{ Chronic disease } \\
\hline Yes & $25(38.5)$ & $16(37.2)$ & 0.896 & $0.948(0.428-2.1)$ \\
\hline No & $40(61.5)$ & $27(62.8)$ & & \\
\hline \multicolumn{5}{|c|}{ Household family members with a chronic disease } \\
\hline Yes & $13(20)$ & $10(23.8)$ & 0.639 & $0.800(0.314-2.037)$ \\
\hline No & $52(80)$ & $32(76.2)$ & & \\
\hline \multicolumn{5}{|c|}{ Using $\mathrm{MoH}^{\mathrm{e}}$ for information } \\
\hline Yes & $42(65.6)$ & $25(58.1)$ & 0.443 & $1.375(0.620-3.046)$ \\
\hline No & $22(34.4)$ & 18 (41.9) & & \\
\hline \multicolumn{5}{|c|}{ Using scientific publications for information } \\
\hline Yes & $19(29.7)$ & $11(25.6)$ & 0.643 & $1.228(0.515-2.932)$ \\
\hline No & $45(70.3)$ & $32(74.4)$ & & \\
\hline \multicolumn{5}{|c|}{ Believe in necessity for vaccination } \\
\hline Yes & $35(53.8)$ & $9(20.9)$ & 0.001 & $4.407(1.825-10.645)$ \\
\hline No & $30(46.2)$ & $34(79.1)$ & & \\
\hline \multicolumn{5}{|l|}{ Duration of working } \\
\hline$\leq 5$ years & 18 (27.7) & $24(55.8)$ & 0.003 & $0.303(0.135-0.682)$ \\
\hline$>5$ years & $47(72.3)$ & $19(44.2)$ & & \\
\hline
\end{tabular}

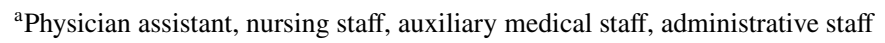

${ }^{\mathrm{b}}$ Physician and physician assistant

${ }^{\mathrm{c}}$ Nursing staff, auxiliary medical staff, administrative staff

${ }^{\mathrm{d}}$ Cohen's $d$-effect size

${ }^{\mathrm{e}}$ Ministry of Health

2016-2017 influenza season. Thus, regular vaccination is low and inconsistent. HCWs who were aware of the necessity of vaccination had higher rates of vaccination, showing a need for education on this point. Because HCWs relied most on the $\mathrm{MoH}$ for information about influenza vaccine recommendations, an active, yearly strategy of outreach by $\mathrm{MoH}$ before influenza season is greatly needed. 


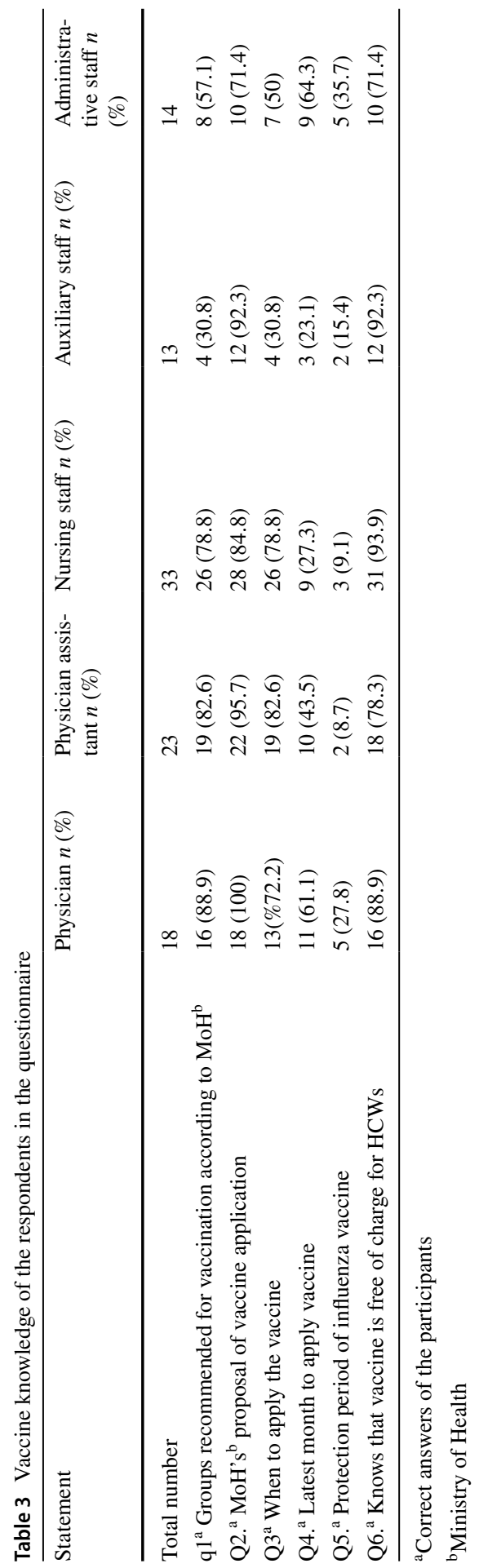


According to the studies of VCRs of HCWs in Turkey, the highest VCRs took place after the 2009 influenza A/H1N1 pandemic, ranging from 12.3 to $76.1 \%$ [10, 16, 18-20]. After that pandemic, VCR for influenza decreased markedly [10]. In 2010 Ulusoy et al. [21] reported that $48.6 \%$ of the physicians and $5.9 \%$ of the nurses received influenza vaccination in a pediatric unit of a hospital. In 2012 Ciblak et al. [22] evaluated 911 family physicians working in two metropolises in Turkey and reported that $45.7 \%$ of family physicians received vaccine annually. According to the statistical agency of Turkey, the nation's influenza VCRs in 2014 fell from 3.3 to $2.6 \%$ in 2016 [23]. In a multicenter study carried out in 2017 in Turkey, the authors found that $7 \%$ of the HCWs received vaccine every year [24]. A European Centre for Disease Prevention and Control technical report from European Union, European Economic Area Member States noted that influenza VCR among HCWs for the 2015-2016, 2016-2017, and 2017-2018 seasons ranged from 15.6 to 63.2\% [25]. We found the overall VCR, and VCR in the 2016-2017 season in our study group to have been lower than in past years reported in Turkey.

Those rates are similar to those in some European countries and far below rates reported from the USA $[3,5,10]$. When we compare our results in Turkey to those in the United States we see some notable differences. We found nurses and physician assistants were considerably less vaccinated than physicians. In the US, during 2017-2018 influenza season, VCR were higher among physicians (96.1\%), but the rates for nurses $(90.5 \%)$ and physician assistants (87.8\%), so the difference among the groups is considerably smaller than in Turkey [5].

According to the vaccine knowledge questionnaire, physicians had the highest level of knowledge about the vaccine, followed by physician assistants. Recently, however, Newcombe et al. [26] found that even pediatricians' knowledge of guidelines and recommendations about influenza vaccination to have been suboptimal in Sydney. A systematic review and meta-regression analysis showed that to increase the vaccination uptake, HCWs' awareness was one of the most effective components, but formal educational interventions (such as presentations, lectures, video projections, meetings) was not [27]. However in a study evaluating the effect of education on awareness, knowledge, and willingness to be vaccinated, the authors found education through audio-visual lectures, one to one discussions, and question and answer sessions by practicing, played an important role in increasing awareness [28].

In Turkey where HCWs relied most commonly on $\mathrm{MoH}$ as their source for information, MoH's data may be the most important data source more broadly because the majority of the hospitals have official connections to MoH. In 2009, Torun et al. [19] found that $35.4 \%$ of the HCWs they studied in Istanbul reported the MoH to be their primary source of knowledge, consistent with our study. The MoH in Turkey is directive and HCWs follow its policies. Since 2009, for its pandemic influenza vaccination program, the Turkish $\mathrm{MoH}$ developed a passive communication strategy, using informative posters. Recently $\mathrm{MoH}$ has posted similar information on its official website [8, 19]. Effective health strategies can be developed by the collaboration between $\mathrm{MoH}$ and hospitals. Policies and activities to encourage vaccines should be brought to the forefront. MoH should ensure that hospitals to organize activities including vaccination days, and HCWs should be specifically tasked 
to increase vaccination rates. Besides providing influenza vaccine free of charge for HCWs, MoH should develop active strategies like informative motivating reminder campaigns about influenza vaccination every year in the hospital and social media can be used to reach all HCWs and community nationwide. These are likely to be more effective than just providing influenza vaccine free of charge with passive notification like website information, posters or presentations.

A review from Turkey about vaccination strategies for HCWs, the reasons for refusing influenza vaccination in HCWs [10] were similar to our findings, that HCWs doubts about the effectiveness/protection capabilities of the vaccine were most common. Different from the reported studies in the same review are some reasons for the rejection of the vaccine in the current study: preferring not to be vaccinated, not liking to receive the vaccine, and lack of familiarity with the vaccine [10]. We suggest that these opinions and preferences may result from the increasing anti-vaccination movement in Turkey. Turkey faces an increasing trend of vaccine refusals-adults for themselves and for their children-due to medical, cultural and religious beliefs. The WHO stated that refusal to vaccinate, despite the availability of the vaccines, is as threatening as the HIV or Ebola diseases. To avoid deaths and severe complications from vaccine-preventable diseases, it is vitally import to increase vaccination rates [15]. HCWs, especially physicians as role models, have an impact on the patient and in all societies; HCWs' vaccine rejections can be expected to have greater impact than refusal by others.

The most common reason to receive the vaccine in our sample was "being at risk". This result is consistent with many studies of HCW vaccination that showed "feel not at risk of getting influenza or its complications" as the main reason for not being vaccinated [17, 29, 30]. Vasilevska et al. [31] reported that consideration of self-protection and desire to protect family and friends appeared the strongest and most consistent driver of HCWs' decisions to accept vaccination.

Lytras et al. [27] reviewed interventions in many countries (such as US,UK, France, Japan, Germany etc.) from many continents to increase seasonal influenza vaccine coverage in HCWs. They found that mandatory vaccination was most effective. The most important difference in practices in Turkey from those discussed in the meta-analysis is the lack of a mandatory vaccination policy. Better on-site access to vaccination, incentives, and education did not have as strong impact on increasing HCWs vaccination as the mandatory vaccination policy did. Implementation of mandatory vaccination is controversial for etnical reasons in Turkey, so the country needs to devise alternative strategies to be as effective as possible. To maintain the specificity of personal preferences, we suggest implementation of a mandatory system of reminders (notification for the vaccine that is due) or recall (notification of the vaccine that is past due) interventions before the influenza season. Because our survey did not include questions about mandatory vaccination, we are unable to discuss the thoughts of HCWs on this issue.

Nearly half of the HCWs in our study had never been vaccinated against influenza. In the 2016-2017 influenza season the VCRs among the HCWs were not only low but declining. Not only our study, but others conducted in Turkey reinforce the lesson that influenza vaccination coverage in Turkey needs to be improved to control influenza morbidity and mortality. In the process of vaccination in Turkey, the most 
obvious difference between Turkey and the US, where VCRs are higher, is lack of mandatory vaccination policy here. Specifically for Turkey, the MoH, professional societies, hospitals and health care authorities should combine common policies with incentives in an active strategy to increase VCRs, especially among HCWs.

The most important limitation of the study is relatively the low number of survey participants as we included only one hospital. Therefore, the results cannot be generalized. However, in our opinion, our study provides useful information about the vaccination rate among healthcare workers. Another limitation is that vaccination status was self-reported and might be subject to recall bias. However, the questionnaire did not include any identifiable data, and participants were assured that all identities would be kept anonymous.

\section{Conclusion}

Considering the reasons for willingness and refusal of the influenza vaccine, accurate risk perception and effective protection from the vaccine should be the most important learning goals in active and annual educational campaigns prior to every influenza season. We suggest that HCWs should be educated with incentivized social campaigns (interactive education about effective protection, necessity, and actual side effects of the vaccine by an authoritative and prominent person in this regard at a hospital-based meeting) during the influenza vaccination season for several days every year instead of the standardized education (posters, presentations, lectures) to increase the awareness and knowledge. HCWs should be the primary target to increase VCRs to protect HCWs and as a means to increase vaccine prevention of influenza nationwide.

Author contributions Shared authorship contributed equally.

Funding None declared.

\section{Compliance with ethical standards}

Conflict of interest All authors declare that they have no conflicts of interest.

\section{References}

1. Biologicals. Influenza. https://www.who.int/biologicals/vaccines/influenza/en/.

2. Kilbourne ED. Influenza pandemics of the 20th century. Emerg Infect Dis. 2006;12(1):9-14.

3. Dini G, Toletone A, Sticchi L, Orsi A, Bragazzi NL, Durando P. Influenza vaccination in healthcare workers: a comprehensive critical appraisal of the literature. Hum Vaccin Immunother. 2018;14(3):772-89.

4. Grohskopf LA, Sokolow LZ, Broder KR, Walter EB, Bresee JS, Fry AM, et al. Prevention and control of seasonal influenza with vaccines: recommendations of the Advisory Committee on Immunization Practices-United States, 2017-18 Influenza season. MMWR Recomm Rep. 2017;66:1-20.

5. Influenza Vaccination Coverage Among Health Care Personnel-CDC. https://www.cdc.gov/ mmwr/volumes/67/wr/mm6738a2.htm. 
6. Dash GP, Fauerbach L, Pfeiffer J, Soule B, Bartley J, Barnard BM, Association for Professionals in Infection C, Epidemiology, et al. APIC position paper: improving health care worker influenza immunization rates. Am J Infect Control. 2004;32:123-5.

7. United States CDC. Prevention and control of influenza: recommendations of the advisory committee on immunization practice (ACIP). MMWR Morbid Mortal Wkly Rep. 2003;52(RR-8)1-36.

8. Mevsimsel Grip. https://grip.gov.tr/tr/saglik-calisani-icin-mevsimsel-grip.

9. Donmez L, Polat HH, Yalcin AN, Oncel S, Turhan O. Influenza vaccination; rates, knowledge and the attitudes of physicians in University Hospital. TurkiyeKlinikleri J Med Sci. 2010;30:48-53.

10. Ozisik L, Tanriovera MD, Altınel S, Unal S. Vaccinating healthcare workers: level of implementation, barriers and proposal for evidence-based policies in Turkey. Hum Vaccin Immunother. 2017;13:1198-206.

11. Black CL, Yue XMPS, Ball SW, Fink R, de Perio MA, et al. Influenza vaccination coverage among health care personnel-United States, 2016-17 influenza season. MMWR Morb Mortal Wkly Rep. 2017;66:1009-15.

12. European Centre for Disease Prevention and Control. Seasonal influenza vaccination in EuropeOverview of vaccination recommendations and coverage rates in the EU Member States for the 2012-13 influenza season. Stockholm: ECDC; 2015. https://ecdc.europa.eu/sites/portal/files/media /en/publications/Publications/Seasonal-influenza-vaccination-Europe-2012-13.pdf.

13. Düzgün MV, Dalgıç AI. Can vaccine rejection, an increasing danger to public health, be prevented? Güncel Pediatri. 2019;17(3):424-34.

14. Tafuri S, Gallone MS, Cappelli MG, Martinelli D, Prato R, Germinario C. Addressing the antivaccination movement and the role of HCWs. Vaccine. 2014;32(38):4860-5.

15. Ten threats to global health in 2019. https://www.who.int/news-room/feature-stories/ten-threats-toglobal-health-in-2019.

16. Savas E, Tanriverdi D. Knowledge, attitudes and anxiety towards influenza A/H1N1 vaccination of healthcare workers in Turkey. BMC Infect Dis. 2010;10:281.

17. Giannattasio A, Mariano M, Romano R, Chiatto F, Liguoro I, Borgia G, et al. Sustained low influenza vaccination in health care workers after H1N1 pandemic: a cross sectional study in an Italian health care setting for at-risk patients. BMC Infect Dis. 2015;15:329.

18. Sevencan F, Ertem M, Ozcullu N, Dorman V, Kubat NK. The evaluation of the opinions and attitudes of healthcare personnel of the province Diyarbakir against influenza A (H1N1) and the vaccination. Hum Vaccin. 2011;7:945-51.

19. Torun SD, Torun F. Vaccination against pandemic influenza A/H1N1 among healthcare workers and reasons for refusing vaccination in Istanbul in last pandemic alert phase. Vaccine. 2010;28:5703-10.

20. Arda B, Durusoy R, Yamazhan T, Sipahi OR, Taşbakan M, Pullukcu H, et al. Did the pandemic have an impact on influenza vaccination attitude? A survey among health care workers. BMC Infect Dis. 2011;11:87.

21. Ulusoy E, Arikan D. Rates of influenza vaccination of healthcare workers working in pediatric units. Turkiye Klinikleri J Nurs Sci. 2010;2(1):11-5.

22. Ciblak MA, Nohutcu N, Gurbuz I, Badur S, Guldal D. Aile hekimliğinde grip ve grip asısı: Bilmek uygulama icin yeterli mi? Türk Aile Hek Derg. 2012;16:157-63.

23. Turkey health care reports. http://www.tuik.gov.tr/Start.do.

24. Tumturk A, Tosun S, Yildız IE, Alay H, Mistanoglu Ozatay D, Mert D, Ugurlu K, et al. Seasonal influenza vaccination coverage: a multicenter cross-sectional study among healthcare workers. Ortadogu Tip Derg. 2020;12(1):113-9.

25. European Centre for Disease Prevention and Control. Seasonal influenza vaccination and antiviral use in EU/EEA Member States-Overview of vaccine recommendations for 2017-2018 and vaccination coverage rates for 2015-2016 and 2016-2017 influenza seasons. Stockholm: ECDC; 2018. https://www.ecdc.europa.eu/en/publications-data/seasonal-influenza-vaccination-antiviral-use-eueea-member-states.

26. Newcombe PJ, Kaur R, Wood N, Seale H, Palasanthiran P, Snelling TL. Paediatrician beliefs and practices around influenza vaccination. J Paediatr Child Health. 2017;53:711-4.

27. Lytras T, Kopsachilis F, Mouratidou E, Papamichail D, Bonovas S. Interventions to increase seasonal influenza vaccine coverage in healthcare workers: a systematic review and meta-regression analysis. Hum Vaccin Immunother. 2016;12(3):671-81.

28. Joshi SV, Chaudhari HR, Chaudhari NA. Effect of education on awareness, knowledge, and willingness to be vaccinated in females of Western India. J Canc Educ. 2020;35:61-8. 
29. Derber CJ, Shankaran S. Health-care worker vaccination for influenza: strategies and controversies. Curr Infect Dis Rep. 2012;14:627-32.

30. Hofmann F, Ferracin C, Marsh G, Dumas R. Influenza vaccination of healthcare workers: a literature review of attitudes and beliefs. Infection. 2006;34:42-7.

31. Vasilevska M, Ku J, Fisman DN. Factors associated with healthcare worker acceptance of vaccination: a systematic review and meta-analysis. Infect Control Hosp Epidemiol. 2014;35(6):699-708.

Publisher's Note Springer Nature remains neutral with regard to jurisdictional claims in published maps and institutional affiliations.

Aysegul Ertugrul MD, is a Paediatric Allergy and Immunology specialist, Health Sciences University Dr. Sami Ulus Maternity and Children Training and Research Hospital, Pediatric Allergy and Clinical Immunology Department, Ankara, Turkey.

Eyup Sari MD, is Paediatrician specialist, Health Sciences University Dr. Sami Ulus Maternity and Children Research and Training Hospital, Department of Pediatrics, Ankara, Turkey.

Nazli Gulenc MD, is Paediatric fellowship, Health Sciences University Dr. Sami Ulus Maternity and Children Research and Training Hospital, Department of Pediatrics, Ankara, Turkey.

Serap Ozmen MD, is Paediatric Allergy and Immunology specialist, Professor of Pediatrics, Health Sciences University Dr. Sami Ulus Maternity and Children Research and Training Hospital, Pediatric Allergy and Clinical Immunology Department, Ankara, Turkey.

\section{Affiliations}

\section{Aysegul Ertugrul $^{1}$ (D) Eyup Sari ${ }^{2} \cdot$ Nazli Gulenc $^{2} \cdot$ Serap Ozmen $^{1}$}

1 Pediatric Allergy and Immunology Department, Health Sciences University Dr. Sami Ulus Maternity and Children Training and Research Hospital, 06080 Altindag, Ankara, Turkey

2 Department of Pediatrics, Health Sciences University Dr. Sami Ulus Maternity and Children Research and Training Hospital, Ankara, Turkey 